\title{
Distribution of benthic polychaetes populations affected by human activities in the west coast of Alexandria, Mediterranean Sea, Egypt
}

\author{
Mohamed M. El-Komi \\ National Institute of Oceanography and Fisheries, Alexandria, Egypt. \\ komimohamed50@yahoo.com
}

\begin{abstract}
Marine benthic communities; in particular the soft bottom polychaetes along the western coast of Alexandria, were studied as part of a monitoring program on the effects of human activities on the ecosystem to determine whether discharges from the wastewater outfalls are affecting the surrounding benthos or nat. These communities are used as indicators of sediment and water quality because they can consume or adsorb the pollutants via feeding processes. Data analyses of benthic communities are commonly used to estimate the changes of complexity of the community structure in the stressed area due to one or more of environmental factors. Soft bottom polychaetes in this area were studied using multivariate and biotic indices, measuring abundance and community composition. Possible significant correlations were determined using multi regression analysis with physical, chemical and biological structure. Twenty six polychaete species belonging to 16 families were identified. The benthic polychaete communities in the study area were dominated by two sedentary forms (Capitellidae, Spionidae) and two errantia forms (Nereidae, Syllidae) besides to unidentified Oligochaeta species. These communities were more dominant in the area near outfalls of El Amom drain, El Dekhelah, Nobaria drain and SUMED area, where their frequencies varied among sites and within sites over the study period. Shannon's index $\left(\mathrm{H}^{\prime}\right)$ varied spatially from 0.49 to 1.76 , Margalef richness index (d) from 0.17 to 1.58 , and evenness index $(\mathrm{J})$ from 0.44 to 0.79 , indicating poor polychaete diversity. High diversity may be attributed to the presence of oppertunitistic species, which are tolerant to high organic matter and chemical residues. The relative frequency of abundant polychaetes can be ranked as follows: Capitella $(15.4 \%)>$ Oligochaeta $(14.9 \%)>$ Polydora $(14.4 \%)>$ Prionospio $(11 \%)>$ Nereis $(10.6 \%)>$ Syllis $(5.4 \%)$. Sedentary polychaetes (Capitellida capitata, Polydora caeca, Prionospio cirrifera) and an errant form (Nereis irrorata) were regularly distributed within stations but their abundance varied between stations. The highest density of species and specimens were recorded at stations $2,4,5,7,8$, and 11, where the tendency of abundance increased from May to July reaching 1092 to $1385 \mathrm{ind} . / \mathrm{m}^{2}$. The increase in polychaete abundance observed in outfall area could be due to the effect of trophic level.
\end{abstract}

Keywords: Polychaetes, human activity, biodiversity, trophic level.

\section{INTRODUCTION}

Untreated pollutants discharges of urban sewage, industrial wastes, land irrigation, fishing and transportation activity in port and near coastal water and the tourist activity are the common anthropogenic disturbances in marine soft-sediment environment that became important problems in recent decades due the increasing beach replacement and land reclamation and growing industry (ICES, 2001). The effects of human activities on near-shore benthic assemblages are still unknown, 
despite a wide increase in demand for marine communities. The long-term environmental impacts of marine extraction are usually site-specific and difficult to assess (Kenny and Rees, 1996; Newell et al., 1998; Desprez, 2000; Boyd et al., 2004).

Transportation in two main harbors (western, El Dekhela), untreated industrial and irrigation discharge from two drains (El Amom and Nobaria) are the most common anthropogenic disturbances in marine soft-bottom environment. Impacts of these factors on benthos can be direct, by smothering or indirect through a permanent change in environmental factors such as turbidity, sediment characteristics and water quality (Jones and Candy, 1981; ICES, 2001). Sediment in this area is often rich in heavy metals, organic matter and nutrient salts, leading to enrichment eutrophication. Degrading of the seabed and benthos following outfalls of different pollutants depends on a variety of factors such as distance from the source, nature of bottom, water circulation action, and depth of water.

Many populations of the benthic invertebrates are immigrating to avoid prolonged exposure to pollution/organic enrichment but some of them are adapted to changes in the environmental conditions. Their occurrence is dependent on the level or limits of tolerance to those environmental variables such the considerable variation over time within the estuarine and coastal sedimentary areas. According to many studies on benthic communities, e.g. Rees et al. (2005); Borja and Muxika (2005), those are used as biological indicators for the impact of human activities. The eutrophication and hypoxia/anoxia (Rumohr, 2005) are acute threats to Baltic biota, and the benthic communities due to sharp environmental gradients and the increasing anthropogenic influences. Organic matter in sediments is a source of food for benthic fauna (Shine, 2005) and over content level can cause reduction in biodiversity indices. The areas located close to the outfall of the main drains are characterized by distinct eutrophication leading to significant increase in species richness and mean abundance of benthic communities of the tolerant species. Quadros et al. (2009) studied monthly Polychaete assemblages and associated environment on intertidal stations along the extremely polluted west coast of India, with silt component of sediment, was increasing with proportionate decrease in clay due to various anthropogenic disturbances.

Research program was conducted along the fishery grounds near Alexandria had been reported in a preliminary study by Steur (1935) with the quantitative determination of bottom fauna, a work that was never tried before in the Eastern Mediterranean. The length of the coast investigated extended from L.E. $29^{\circ} 40^{\prime}$ to the mouth of the Nile at Rosetta at L.E. $30^{\circ} 20^{\prime}$, covering about 150 stations. On the other hand, Fauvel (1937) identified several polychaete species among the bulk of bottom fauna that had been collected during 1933 by Steur survey.

The discharged sewage into seawater increased the amount of small particles suspended in the water column which contributed large amounts of nutrients leading to eutrophication. Its impacts are more significant in the semi enclosed areas, where the benthic invertebrates communities have degraded, algal beds disappeared and diseased fish became more prevalent. Fertilizers used on land are washed into the seawater via rivers and streams. High nutrient concentrations cause phytoplankton bloom "red tides" that grow over bottom invertebrates communities and eventually smothers them to death. Contamination levels and the main press of anthropogenic impact increased in the enriched seas, coasts, estuaries and shelf waters, i.e. in the zones of high bio productivity and marine living resources that is extremely ecologically alarming. 
This study assessed the state of polychaete assemblages at sites affected by different sources of pollutants. Bottom sediments were collected from impact sites to estimate benthic community's abundance; species richness and evenness of polychaetes, as well as the overall polychaete assemblage's structure that was compared between localities over time.

\section{MATERIALS AND METHODS}

\section{Study area}

The selected sites represented the different habitats along the inshore as shown in Table (1) which can be distinguished into 3 stressed regions:

\section{a) High stressed region}

Site at one location within the western harbor of Alexandria (St.1). This site is greatly influenced by the transportation to and out of the harbor (Fig. 1).

Three locations within El Mex Bay at about $0.5 \mathrm{~km}, 1.5 \mathrm{~km}$ and $2 \mathrm{~km}$ away from El Amom outfall drain (Sts.2, 3, 4). Sites at two locations in the El Dekhelah harbor at about one $\mathrm{km}$ and $2 \mathrm{~km}$ (Sts.5, 6). These sites have large shipping containers and are parallel to the highly metropolitan region to the west of Alexandria.

\section{b) Moderate stressed region}

Sites at four locations at about $0.5 \mathrm{~km}, 1.5 \mathrm{~km}, 2 \mathrm{~km}$ and $3 \mathrm{~km}$ away the outfall of Nobaria drain (Sts.7, 8, 9, 10), with land use ranging from freshwater and irrigation drainage water (Fig. 1).

\section{c) Less stressed region}

\section{1- SUMED area}

Sites at two locations within SUMED area where the petroleum pipe line extends from the Red Sea to the Mediterranean Sea, located at about $2 \mathrm{~km}$ and $3 \mathrm{~km}$ from the shoreline (Sts.11, 12) (Fig. 1).

\section{2- Tourist area}

Sites at 4 locations are located along $40 \mathrm{~km}$ to the west of SUMED area that are highly affected by the restoration activities where St.13 (located at about $4 \mathrm{~km}$ ), Sts.14, 15 (within Marakia village) and St.16 within Marbella village. These sites are considered oligotrophic (Fig. 1) that are located far from the sources of pollution.

\section{Sampling}

The sampling plan included sixteen stations located along eight transects spaced $500 \mathrm{~m}$ to $5 \mathrm{~km}$ apart, with sampling sites at 2 to $25 \mathrm{~m}$ (Fig. 1 and Table 1) where the bottom sediments were sampled bimonthly during the period from March 2005 to January 2006 by using a modified Van Veen grab with a sampling surface of $0.023 \mathrm{~m}^{2}$. Grab sample was placed in containers with $5-10 \%$ formalin/seawater and transferred for the laboratory analysis.

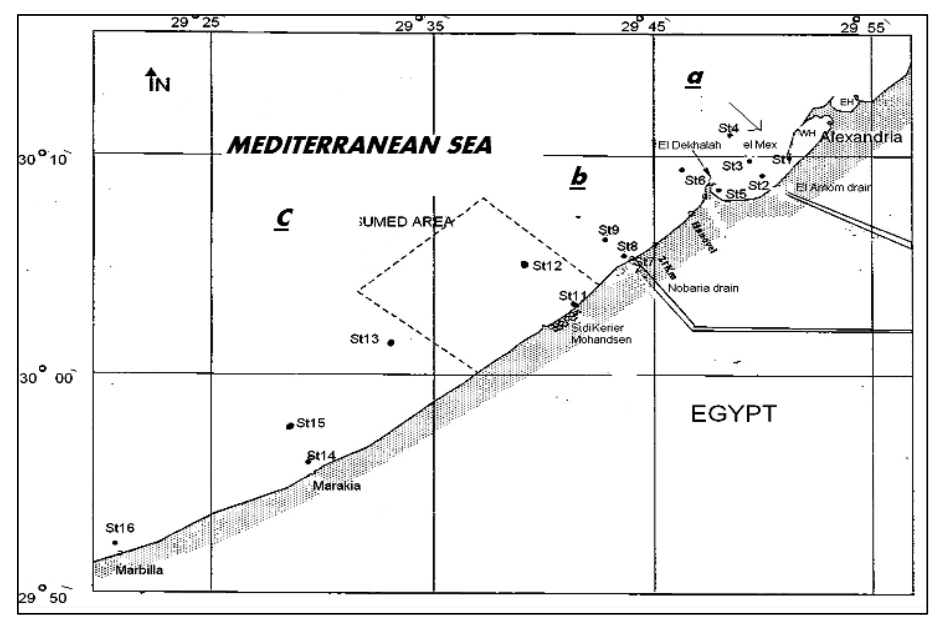

Fig. 1: Area of study and sampling stations, west of Alexandria 
Table 1: Sixteen sites were selected to represent the different habitats along the inshore.

\begin{tabular}{|c|l|l|l|}
\hline Station & \multicolumn{1}{|c|}{ Bottom habitat } & \multicolumn{1}{|c|}{ locality } & depth \\
\hline 1 & Sludge/shells & out side the western harbor of Alexandria & $5 \mathrm{~m}$ \\
\hline 2 & Sludge/plant fragments & Inside El Mex Bay close to El Amom drain & $6 \mathrm{~m}$ \\
3 & Sludge/plant fragments & middle El Mex Bey-El Amom drain & $12 \mathrm{~m}$ \\
4 & coarse sand & out side El Mex Bay-El Amom drain & $10 \mathrm{~m}$ \\
\hline 5 & fine/coarse sand & close to Al Agamy beach,dense,urban area & $13 \mathrm{~m}$ \\
6 & coarse sand & out side Al Agamy beach & $14 \mathrm{~m}$ \\
\hline 7 & Sludge/plant fragments & close to El Nobaria drain & $2.5 \mathrm{~m}$ \\
8 & fine/coarse sand & out side El Nobaria drain & $4 \mathrm{~m}$ \\
9 & fine/coarse sand & out side El Nobaria drain & $9 \mathrm{~m}$ \\
10 & fine/coarse sand & out side El Nobaria drain & $12 \mathrm{~m}$ \\
\hline 11 & fine/calcareous sand & SUMED oil transport area, km 21 Sidi Kerier & $2 \mathrm{~m}$ \\
12 & fine/calcareous sand & SUMED oil transport area, km 21 Sidi Kerier & $25 \mathrm{~m}$ \\
\hline 13 & fine/calcareous sand & lies in between Sts. 12, 15 & $22 \mathrm{~m}$ \\
\hline 14 & fine/calcareous sand & in front Marakia resort beach & $2 \mathrm{~m}$ \\
15 & fine/calcareous sand & out side the area of Marakia resort beach & $20 \mathrm{~m}$ \\
\hline 16 & fine/calcareous sand & in front Marbilla resort beach & $2 \mathrm{~m}$ \\
\hline
\end{tabular}

\section{Sorting and identification}

In the laboratory, each sample was washed and sieved through a series of sieves from 0.01 to $0.5 \mathrm{~mm}$ and the residue was placed in small Petri dishes and all sorted animals were identified to the lowest possible taxonomic level by using a stereomicroscope $(20 \mathrm{x}, 40 \mathrm{x})$. Oligochaetes were not identified to lower level, while polychaetes were identified to lower taxa (26 species belonging to 16 families). The species list of polychaete populations used for the present study was performed at families, generic and species levels to provide database as a reference collection for polychaetes in the area of study. The abundance of the bottom polychaetes was expressed as the number of individuals $/ \mathrm{m}^{2}$.

\section{Data analysis}

Statistical analyses of the polychaete fauna of the different localities were performed using standard methods:

a- Multivariate analysis using Primer v5 package (Clarke and Gorley, 2001).

b- Bray-Curtis similarity matrices (Bray-Curtis, 1957).

c- Species diversity indices (Shannon-Weiver, Evenness, Richness) using Primer v5 package (Clarke and Gorley, 2001).

d- Swartz's dominance index (comprised $75 \%$ of the total sample abundance) according to PTI 1993 as cited by Laetz, 1998).

e- The analysis of variance (ANOVA) were computed by using simple linear regression between the variables of polychaetes at sampling sites (abundance, biomass, $\mathrm{H}^{\prime}$, number of species, main benthos groups) and physio-chemical variables of seawater and sediments $\left(\mathrm{Ni}, \mathrm{Mn}, \mathrm{Fe}, \mathrm{Cu}, \mathrm{Zn}, \mathrm{Pb}, \mathrm{Cd}, \mathrm{NH}_{3}, \mathrm{NO}_{3}, \mathrm{NO}_{2}\right.$, $\mathrm{PO}_{4}, \mathrm{SiO}_{4}, \mathrm{~S} \%$ o, temperature, $\mathrm{pH}$ as listed in the final Report, NIOF, 2006).

\section{RESULTS}

\section{The benthic polychaetes environment}

The sediments along the coastal area from the first sector namely Western Harbour of Alexandria to Marbilla beach was highly polluted by eutrophication parameters of the sediment particularly in the stressed region in front of Al Amom and Nobaria drains. Bottom features, included main sedimentary structures composed mainly of sludge and fragments of shells and sea grass particularly at sites located close to the opening of out flow of the El Amom and El Nobaria drains. The sediment bottom surface was slightly polluted and 
composed mainly of calcareous fine sands and coarse sands that were widely distributed at the shallow and deeper depths of most sites. The study area was characterized by a prevalence of high transparency of sea water at the sites located at most western sectors from St.11 to St.16, reaching $12 \mathrm{~m}$ depth at deeper depths, moderately at stations apart of the out flow of drainage water from El Amom and El Nobaria drains to poor values $(0.3-0.5 \mathrm{~m})$ at stations close to opening of the above drains. Temperature of surface sea water showed very low variations among different stations, while it greatly varied seasonally from $17^{\circ} \mathrm{C}$ in January and March to $30^{\circ} \mathrm{C}$ in July and $24-27^{\circ} \mathrm{C}$ during May, September, and November. The following chemical parameters were taken from the final report of NIOF (2006). The value of $\mathrm{pH}$ of water column was low (7.7) at St. 2 and ranged from 8.1 to 8.3 at most of the rest sites. The dissolved oxygen content in sea water was low $(4.6 \mathrm{mg} / \mathrm{l})$ at highly stressed region (El Mex Bay and front El Nobaria drain) ranged from 7.5 to $9.3 \mathrm{mg} / \mathrm{l}$ at most of the rest stations. The heavy metals content in bottom sediments showed wide variations at the different sampling sites as following: $\mathrm{Ni}, \mathrm{Mn}, \mathrm{Fe}, \mathrm{Cu}, \mathrm{Cd}, \mathrm{Pd}$ in sediments were respectively ranging between 12.4 to $29.4 \mathrm{ug} / \mathrm{g}, 43$ to $253 \mathrm{ug} / \mathrm{g}, 514$ to $2310 \mathrm{ug} / \mathrm{g}, 20.7$ to $69.6 \mathrm{ug} / \mathrm{g}, 0.8$ to $4.1 \mathrm{ug} / \mathrm{g}$ and 6.8 to $51.9 \mathrm{ug} / \mathrm{g}$. In general, the heavy metals content in sediments increased relatively in the first three sites namely; St.1, St.2 and St.3.

\section{Polychaetes species structure}

Twenty six polychaete species $(12,272$ individuals) belonging to 16 families were identified (Table 2). The benthic polychaete communities in the study area were dominated by two sedentary forms (Capitellidae, Spionidae) and two errant forms (Nereidae, Syllidae) besides to unidentified oligochaete species.

Table 2: List of polychaete species recorded at the different sampling sites at the western coastal waters of Alexadria during March 2005 to January 2006.

\begin{tabular}{|c|c|}
\hline Annelida : & Genus Onuphis Audouin et M. Edwards, 1834 \\
\hline Oligochaeta : & Onuphis eremita Audouin et M. Edwards, 1834 \\
\hline Errantia polychaetes & Genus Diopatra Audouin et M. Edwards 1834 \\
\hline 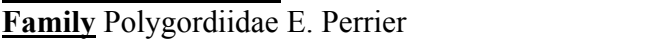 & Diopatra neapolitana Delle Chiaje 1825 \\
\hline Genus Polygordius Schneider, 1868 & Genus Lumbriconereis Blainnville Lumbriconereis \\
\hline $\begin{array}{l}\text { Polygordius neapolitanus Faripont } 1887 \\
\text { Genus Saccocirrus Bobretzky, } 1871\end{array}$ & laterilli Aud. M. Edward \\
\hline Saccocirrus major Pierantoni Bobretzky, 1907 & \\
\hline Family Aphroditidae Savigny, 1820 & Sedentary polychaetes \\
\hline 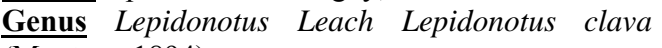 & $\overline{\text { Family Spionidae Sars, }} 1861$ \\
\hline 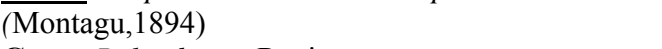 & 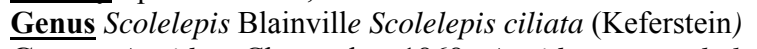 \\
\hline $\begin{array}{l}\text { Genus Polyodontes Renier } \\
\text { Polyodontes maxillosus Ranzani }\end{array}$ & $\begin{array}{l}\text { Genus Aonides Claparede, } 1868 \text { Aonides oxycephala } \\
\text { (Sars, 1862) }\end{array}$ \\
\hline Family Phyllodocidae Grube, 1848 & Genus Polydora Bosc Polydora caeca (Oersted 1843) \\
\hline Genus Eulalia Oersted 1843 & $\overline{\text { Genus }}$ Prionospio Malmgren 1868 \\
\hline$\overline{\text { Eulalia viridis (Muller, 1855) }}$ & 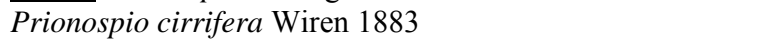 \\
\hline Genus Maupasia Viguier, 1886 & Family Magelonidae Cunningham et Ramage 1888 \\
\hline Maupasia isochaeta (Reibisch,1895) & Genus Magelona F. Muller 1855 \\
\hline Family Syllidae Grube, 1863 & Magelona papillicornis F. Muller 1855 \\
\hline Genus Syllis Savigny, 1820 & Family Cirratulidae Carus, 1840 \\
\hline Syllis gracilis Grube, 1863 & 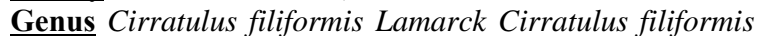 \\
\hline Family Nereidae Quatrefages & $\overline{\text { Keferstein }}$ \\
\hline Genus Nereis Cuvi & Family Opheliidae Grube 1848 \\
\hline irrorata (Molmgren, 1867) & $\overline{\text { Genus }}$ Armandia Filippi Armandia \\
\hline Nenhthrdidae Gr & Kekenthal 1887 \\
\hline Genus Nephys Cuvier, 1830 Nephthyis hombergii & Family Capitellidae Grube 1848 \\
\hline 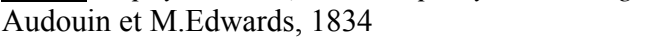 & $\overline{\text { Genus }}$ Capitella Blaniville Capitella capitata (Fabricius, \\
\hline Family Glyceridae Grube, 1863 & $\overline{1780)}$ \\
\hline Genus Glycera Savigny, 1820 Glycera convoluta & 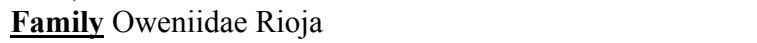 \\
\hline Keferstein, 1862 & Genus Owenia Delle Chiaje Owenia fusiformis Delle \\
\hline Genus Goniada Audouin et M. Edwards 1843 & $\overline{\text { Chiaje }}$ \\
\hline Goniada norvegica Oersted 1843 & Family Sabellidae Malmgren \\
\hline Family Eunicidae Grube, 1863 & Genus Sabella Linne Sabella pavonina Savigny 1820 \\
\hline $\begin{array}{l}\text { Genus Eunice Cuvier } 1830 \text { Eunice vittata (Delle } \\
\text { Chiaje, 1825) }\end{array}$ & $\begin{array}{l}\overline{\text { Genus }} \\
\text { (Montagu, } 1804 \text { ) }\end{array}$ \\
\hline
\end{tabular}




\section{Geographical Distribution of polychaetes species, according to Fauvel (1923, 1927, 1937). \\ Errant polychaetes}

Polygordius neapolitanus**; Mediterranean (Naples).

Saccocirrus majori; Mediterranean, Villefranche, Naples.

Lepidonotus clava; Under stones in the littoral zone- Channel, Mediterranean, Atlantic Cape de Bonne- Esperance, Indian Ocean.

Polyodontes maxillosus**; Atlantic (Santander), Mediterranean, Adriatic Eulalia viridis; North Sea, Channel, Atlantic, Mediterranean, Indian, Pacific Maupasia

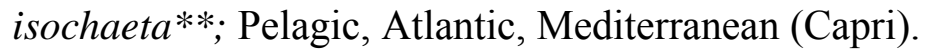

Syllis gracilis; Channel, Atlantic, Mediterranean, Indian Ocean, Pacific.

Nereis irrorata; North Sea, Channel, Atlantic, Mediterranean, Adriatic Sea-Arctic Sea. Nephthyis hombergii; North Sea, Channel, Atlantic, Mediterranean, Adriatic, Suez Canal.

Glycera convolute; Channel, Atlantic, Mediterranean, Red Sea.

Goniada norvegica**; Muddy sand, Dredging, North Sea, Atlantic, (Belle Isle, Cape Finisterre), Mediterranean, (coast of Sicily).

Eunice vittata; Dredging coastal Atlantic (Brest, Concarrneau, Glenaus)Mediterranean - Pacific (Japan, Hawaii, Australia) Onuphis eremite; Atlantic (La Rochelle, Noirmutier, Santander, dimensions of the Sahara, the Dohomey, Congo); Mediterranean (Naples), Adriatic Sea - Indian Ocean (Madagascar, Ceylon).

Diopatra neapolitana; Atlantic (Archon, Saint-ean-de-Luz, Santander; Coast of Western Africa; Mediterranean (Naples, Palermo) Indian-Ocean, Red Sea.

Lumbriconereis latreilli; Arctic seas; Atlantic (Bay of Biscay, the Channel entry); Mediterranean (Algeria, Monaco), Japan, Patchily Gulf.

Sedentary polychaetes

Scolelepis ciliata**; North Sea, Channel, (Sand-Cast, Anise-St-Martin, Cherbourg, northern coast Bretaque).

Aonides oxycephala**; North Sea, Channel, Atlantic (coast of Ireland Crosio, Santander, America), Mediterranean (Port-Venders).

Polydora caeca**; North Sea, Channel, Atlantic, Mediterranean, Arctic-Sea Prionospio malmgren**; North Sea, Atlantic (Ireland, Concarneau, Glenna's, Madre) Mediterranean (Naples, Marseille).

Magelona papillicornis; North Sea, Channel, Atlantic, Mediterranean (Naples) Cirratulus filiformis**; Mediterranean (Naples, Marseilles, Malaga).

Armandia polyophthalma; Mediterranean, Atlantic, Channel.

Capitella capitata; North Sea, Channel, Atlantic, Mediterranean, Sea-Arctic, Black Sea.

Owenia fusiformis; North Sea, Channel, Atlantic, Mediterranean, South Atlantic, Indian Ocean, Pacific.

Sabella pavonina; North Sea, Channel, Atlantic, Mediterranean.

Bispira volutacornis; Channel, Atlantic, Mediterranean.

$\dagger^{* *}=$ new record in Alexandria coastal area.

\section{Temporal abundance}

As listed in Table (3) and illustrated histographically in Fig. 2, a high density was recorded during May (average $1024 \mathrm{ind} . / \mathrm{m}^{2}$ ) and November (average 1215 ind $/ \mathrm{m}^{2}$ ). The abundance variations in space can be attributed to changes in the habitats as the structure of bottom sediments, depth of water, quality of water ( $\mathrm{S} \% 0$, oxygen content, $\mathrm{pH}$, pollutants enrichment, etc.). In this survey the polluted areas contributed the well diverse and evenness values recorded rather than those of less 
polluted areas. These communities can be persisted and modified to types of disturbances induced by domestic sewage, heavy metals, chemical residue discharges from waste industrial, irrigation drainage, and fishing and navigation activities.

A tendency of abundance was relatively low in March (424 ind. $/ \mathrm{m}^{2}$ as total average recorded at sampling sites via the period of collections) and maximal in number in May and November, attaining 1024 and 1215 ind. $/ \mathrm{m}^{2}$, respectively. The average density during period of collections varied between 424 to $1215 \mathrm{ind} . / \mathrm{m}^{2}$ with an annual average 765 ind. $/ \mathrm{m}^{2}$ (st.d. \pm 204 ) its tendency of increasing is related to temperature of water. The errant polychaetes (Nereis irrorata) varied between and within stations. The highest density of species and specimens were recorded at Sts.2, $4,7,11$. A tendency of abundance increased from May to July.

Table 3: Bimonthly changes in average density of the recorded polychaetes species during the study period.

\begin{tabular}{|c|c|c|c|c|c|}
\hline Mar & Av.no & $\%$ & may & Av.no. & $\%$ \\
\hline Capitella capitata & 168 & 39.6 & Nereis irrorata & 478 & 46.7 \\
\hline Prionospio cirrifera & 122 & 28.8 & Oligochaeta & 171 & 16.7 \\
\hline Nereis irrorata & 50 & 11.7 & Capitella capitata & 162 & 15.8 \\
\hline Oligochaeta & 27 & 6.3 & Polydora caeca & 71 & 6.9 \\
\hline Owenia fusiformis & 12 & 2.7 & Nephthys homborgii & 49 & 4.7 \\
\hline Syllis gracilis & 12 & 2.7 & Prionospis cirrifera & 45 & 4.4 \\
\hline Glycera convoluta & 12 & 2.7 & Syllis gracilis & 26 & 2.5 \\
\hline Polydora caeca & 8 & 1.8 & Megelona papillicornis & 13 & 1.3 \\
\hline Lepidonotus clava & 8 & 1.8 & Glycera convoluta & 10 & 0.9 \\
\hline \multirow[t]{2}{*}{ Nephthys hombergii } & 8 & 1.8 & & & \\
\hline & 424 & 100 & & 1024 & 100 \\
\hline Jul & Av.no & $\%$ & Sep & Av.no. & $\%$ \\
\hline Capitella capitata & 174 & 22.713 .9 & Oligochaeta & 207 & 26.7 \\
\hline Prionospio cirrifera & 106 & 11.4 & Cirratulus filiformis & 107 & 13. \\
\hline Polygordius sp. & 87 & 10.6 & Onuphis eremita & 94 & 12.1 \\
\hline Syllis gracilis & 81 & 9.2 & Diopatra neapolitana & 91 & 11.7 \\
\hline Saccocirrus major & 70 & 8.1 & Nephthys homborgii & 55 & 7.1 \\
\hline Eunice vittata & 62 & & Lumbriconereis latreilli & 45 & 5.8 \\
\hline Armandia & & 7.7 & Lepidonotus clava & 45 & 5.8 \\
\hline pulyophthalma & 59 & 6.2 & Aonides oxycephala Glycera & 29 & 3.7 \\
\hline Cirratulus filiformis & 48 & 3.3 & convoluta & 26 & 3.3 \\
\hline Glycera convoluta & 25 & 2.6 & Sabella pavonina & 16 & 2.1 \\
\hline Nephthys homborgii & 20 & 1.5 & Syllis gracilis & 16 & 2.1 \\
\hline Polydontes maxillosus & 11 & 1.5 & Capitella capitata & 13 & 1.7 \\
\hline Lumbriconereis latreilli & 11 & 1.1 & Eulalia viridis & 10 & 1.2 \\
\hline Eulalia viridis & 8 & 0.4 & Armandia pulyophthalma & 10 & 1.2 \\
\hline \multirow[t]{3}{*}{ Maupasia isochaeta } & 3 & & Nereis irrorata & 7 & 0.8 \\
\hline & & & Magelona papillicornis & 7 & 0.8 \\
\hline & 764 & 100 & & 775 & 100 \\
\hline Nov & Av.no & $\%$ & Jan & Av.no & $\%$ \\
\hline Polydora caeca & 640 & 53 & Oligochaeta & 189 & 24.7 \\
\hline Prionospio cirrifera & 281 & 23 & Capitella capitata & 165 & 21.6 \\
\hline Oligochaeta & 110 & 9 & Syllis gracilis & 105 & 13.7 \\
\hline Capitella capitata & 71 & 6 & Glycera convoluta & 60 & 7.8 \\
\hline Glycera convoluta & 45 & 4 & Prionospio cirrifera & 51 & 6.7 \\
\hline Armandia & & & Scolelepis ciliata & 42 & 5.5 \\
\hline pulyophthalma & 23 & 2 & Saccocirrus major & 36 & 4.7 \\
\hline Syllis gracilis & 13 & 1 & Armandia pulyophthalma & 33 & 4.3 \\
\hline Cirratulus filiformis & 10 & 1 & Lepidonotus clava & 18 & 2.4 \\
\hline Nephthys homborgii & 10 & 1 & Nephthys homborgii & 15 & 2.0 \\
\hline Bispira volutacornis & 7 & 1 & Onuphis eremita & 10 & 2.0 \\
\hline \multirow[t]{5}{*}{ Eunice vittata } & 7 & 1 & Polydora caeca & 12 & 1.6 \\
\hline & & & Nereis irrorata & 9 & 1.2 \\
\hline & & & Goniada norvegica & 9 & 1.2 \\
\hline & & & Cirratulus filiformis & 6 & 0.8 \\
\hline & 1215 & 100 & & 765 & 100 \\
\hline
\end{tabular}



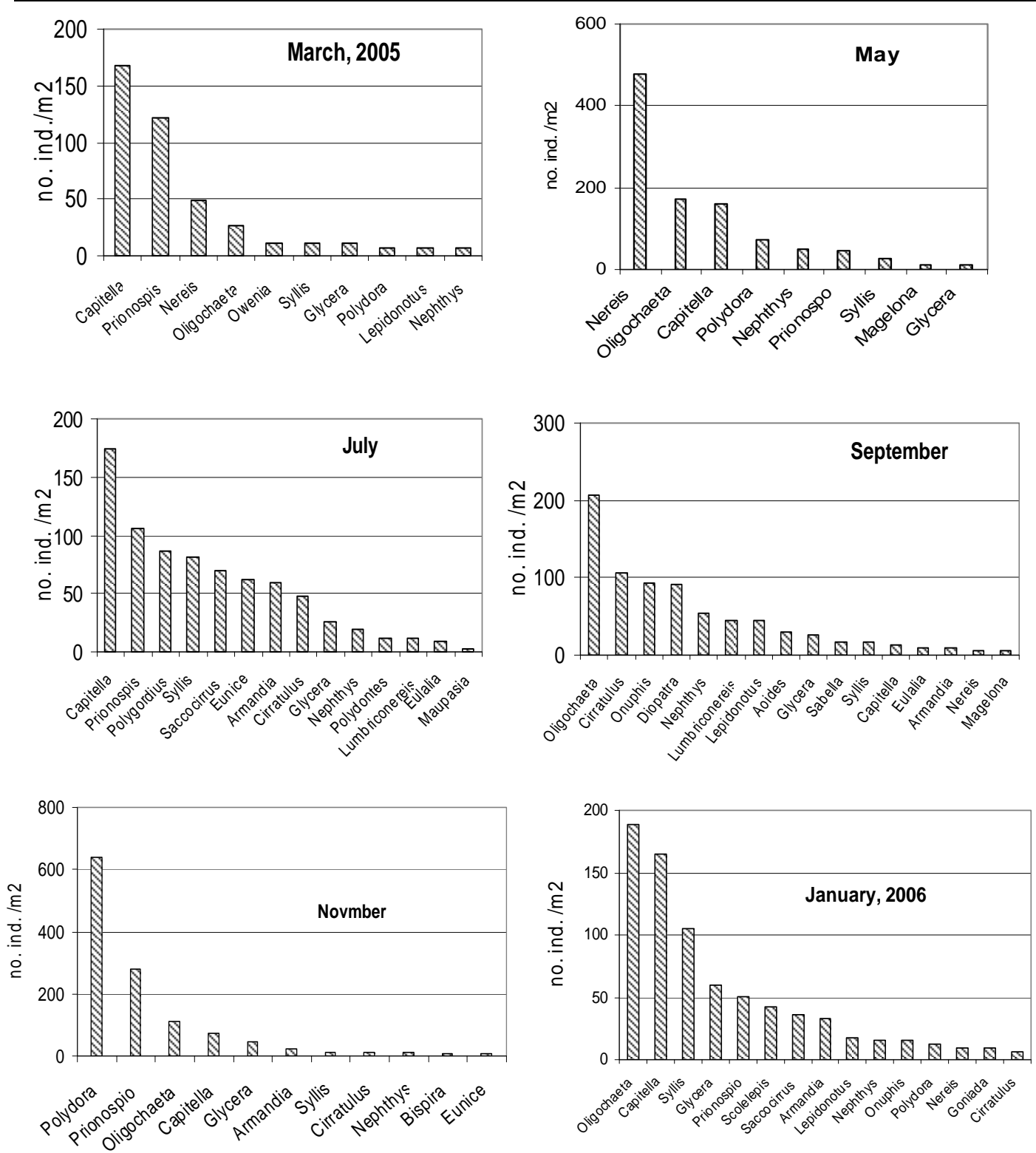

Fig. 2: Bimonthly changes in average density of the recorded oligochaetes and polychaetes species during the study period.

Abundance of the four numerically dominant species among the period of collections and at each station is listed in Table (4) which was greatly varied among times and between sites. Total average abundances of organisms ranged from low number (424 ind. $\left./ \mathrm{m}^{2}\right)$ in March to the highest density $\left(1215 \mathrm{ind} . / \mathrm{m}^{2}\right)$ in November. The sampling sites had more or less similar community compositions over the time; also the percent of organisms in each of the major taxonomic groups varied for each site. In March, each of Prionospio cirrifera and Capitella capitata showed high percentage, attaining $29 \%$ and $40 \%$ respectively. They contributed $56 \%$ at St. 4 and $18 \%$ at St. 5. On the other hand, Nereis irrorata and Oligochaeta had significant abundance in May rather than other months reaching $47 \%$ and $17 \%$ of the total average abundance. 
Table 4: Abundance of the four numerical dominant species at each site for each collection period.

\begin{tabular}{|c|c|c|c|c|}
\hline $\begin{array}{l}\text { Period of } \\
\text { collection }\end{array}$ & Major abundance species & $\%$ & $\%$ at each site & $\begin{array}{r}\text { Total average } \\
\text { Abundance }\end{array}$ \\
\hline March & $\begin{array}{l}\text { Capitella capitata } \\
\text { Prionospio cirrifera } \\
\text { Nereis irrorata } \\
\text { Oligochaeta }\end{array}$ & $\begin{array}{l}40 \\
29 \\
12 \\
6.3\end{array}$ & $\begin{array}{ll}\text { St. } 4 & 56 \% \\
\text { St. } 5 & 8 \% \\
\text { St. } 1 & 8.1 \%\end{array}$ & $\begin{array}{l}424 \\
\text { ind./ } \mathrm{m}^{2}\end{array}$ \\
\hline May & $\begin{array}{l}\text { Nereis irrorata } \\
\text { Oligochaeta } \\
\text { Capitella capitata } \\
\text { Polydora caeca }\end{array}$ & $\begin{array}{l}47 \\
17 \\
15 \\
6.9\end{array}$ & $\begin{array}{lc}\text { St. } 7 & 30 \% \\
\text { St. } 6 & 16 \% \\
\text { St. } 3 & 15 \%\end{array}$ & $\begin{array}{l}1024 \\
\text { ind./ } \mathrm{m}^{2}\end{array}$ \\
\hline July & $\begin{array}{l}\text { Capitella capitata } \\
\text { Prionospis cirrifera } \\
\text { Polygordius } \\
\text { neapolitanus Syllis gracilis }\end{array}$ & $\begin{array}{l}22.7 \\
13.9 \\
11.4 \\
10.6\end{array}$ & $\begin{array}{ll}\text { St. } 5 & 18 \% \\
\text { St. } 8 & 16 \% \\
\text { St. } 9 & 15 \% \\
\text { St. } 4 & 11 \% \\
\end{array}$ & $\begin{array}{l}764 \\
\text { ind./ } \mathrm{m}^{2}\end{array}$ \\
\hline September & $\begin{array}{l}\text { Oligochaeta } \\
\text { Cirratulus filiformis } \\
\text { Diopatra neapolitana } \\
\text { Onuphis eremita } \\
\end{array}$ & $\begin{array}{l}28.1 \\
14.5 \\
12.3 \\
12.7\end{array}$ & $\begin{array}{ll}\text { St. } 1 & 23.7 \% \\
\text { St.5 } & 10.1 \% \\
\text { St. } 9 & 18 \%\end{array}$ & $\begin{array}{l}776 \\
\text { ind. } / \mathrm{m}^{2}\end{array}$ \\
\hline November & $\begin{array}{l}\text { Polydora caeca } \\
\text { Prionospis cirrifera } \\
\text { Oligochaeta } \\
\text { Capitella capitata }\end{array}$ & $\begin{array}{l}52.6 \\
23.1 \\
9 \\
5.8 \\
\end{array}$ & $\begin{array}{l}\text { St. } 11 \quad 39 \% \\
\text { St. } 8 \quad 13 \% \\
\text { St. } 14 \quad 10 \%\end{array}$ & $\begin{array}{l}1215 \\
\text { ind./ } \mathrm{m}^{2}\end{array}$ \\
\hline January & $\begin{array}{l}\text { Oligochaeta } \\
\text { Capitella capitata } \\
\text { Syllis gracilis } \\
\text { Glycera convoluta }\end{array}$ & $\begin{array}{l}24.7 \\
21.6 \\
13.7 \\
7.8\end{array}$ & $\begin{array}{l}\text { St. } 16 \\
22.8 \% \\
\text { St. } 11 \\
10.6 \% \\
\text { St. } 611 \% \\
\text { St. } 510 \%\end{array}$ & $\begin{array}{l}765 \\
\text { ind. } / \mathrm{m}^{2}\end{array}$ \\
\hline
\end{tabular}

\section{Regional Abundance}

The data analysis of the regional distribution showed 5 dominant species namely; the sedentary polychaetes (Capitellida capitata, Polydora caeca, Prionospis cirrifera) and two errant polychaetes (Nereis irrorata, Sillys gracilis) and an unidentified oligochaete so they varied between and within stations. Oligochaetes and polychaetes species were regularly distributed within stations but their abundance varied between stations (as seen in Table 5 and Fig. 3). The highest density of species and specimens were recorded at Sts. 2, 4, 5, 7, 9 and 11, where they were significantly affected by sources of pollutants from El Amom and El Nobaria drains. The average density at different sites ranged from 164 to $1396 \mathrm{ind} . / \mathrm{m}^{2}$ with an annual average of 770 ind. $/ \mathrm{m}^{2}$ (st.d. \pm 442.5 ). The data analysis of the regional distribution of the 5 dominant species indicated that the sedentary polychaetes (Capitellida capitata, Polydora caeca, Prionospio cirrifera) were regularly distributed within stations but their abundance varied between stations (Table 5).

Oligochaetes, were dominant in all coastal areas of study influenced by sewage (El Amom drain), fresh water irrigation (El Nobaria drain) at different depths (3-25 $\mathrm{m})$ as well as in mesohaline areas (Sts. 2, 5, 7) yielding respectively 448, 245 and 202 ind. $/ \mathrm{m}^{2}$ as shown in Table (3), and Fig. 3.

Oligochaeta appears more productive at St.6 (2024 org. $\left./ \mathrm{m}^{2}\right)$, than at St.7 (488 org. $/ \mathrm{m}^{2}$ ) and St.2 (414 org. $/ \mathrm{m}^{2}$ ) during the summer and autumn due to the low chloride content of water in the south east part of El Mex Bay. The average annual number of Oligochaeta counted 396 org. $/ \mathrm{m}^{2}$ that contributed $28.7 \%$ of the total benthos but it showed a very low biomass $\left(0.36 \mathrm{~g} / \mathrm{m}^{2}\right)$.

\section{Polychaetes including the following species}

Capitella capitata, present by great number co-dominant with oligochaetes at all study areas and less frequent in tourist area. It appears more productive, as estimated by the average total specimens collected via the period of collections, at Sts. 2, 4, 5, 9 
counting respectively $210,427,252$, and $301 \mathrm{ind} . / \mathrm{m}^{2}$ at sampling sites. It was recorded in great numbers in May and July due to the water temperature and low water salinity. Its density was high during March (168 ind.$/ \mathrm{m}^{2}$ contributing $39.6 \%$ of total averages of oligochaetes and polychaetes) being maximal at St. 4, contributing $56 \%$ of the total density during March, reached 161 ind.$/ \mathrm{m}^{2}$ in May contributing $15.8 \%\left(161 \mathrm{ind} . / \mathrm{m}^{2}\right)$ appearing relatively high at St. 3 by $15 \%$ of the total density during May, while in July it reached $174 \mathrm{ind} . / \mathrm{m}^{2}$ representing $22.7 \%$ of the total average and $18 \%$ of the total density during July at St. 3 .

Polydora caeca, less frequent at the study areas and recorded only at St.2 during March, May, and January, yielding 84,924 and $168 \mathrm{ind} . / \mathrm{m}^{2}$ respectively. It was more frequent during November counting 168 at Sts. 2, 9.10 and was maximal at Sts. 11 and 14, reaching 6090 and 1568 ind. $/ \mathrm{m}^{2}$, respectively. It was recorded at different depths to $25 \mathrm{~m}$ and at different water quality; so it can tolerate wide variations in the stressed environment area with pollutants.

Table 5: Regional distribution of average of individuals of polychaetes and oligochaetes during the study period. Bottom sediments structure: Sludge $=\mathrm{sl}$. Coarse sand=c.s. Fine sand=f.s.

Calcareous fine sand $=$ ca.f.s.

\begin{tabular}{|c|c|c|c|c|c|c|c|}
\hline St.1 $5 m$, sl. & No. & St.5 13m, f.s., c,s. & No. & St.8 $4 m$, f.s. & No. & St.11 2m, ca.f.s. & No. \\
\hline Oligochaeta & 56 & Oligochaeta & 245 & Prionospio cirrifera & 357 & Oligochaeta & 91 \\
\hline Prionospio cirrifera & 42 & Prionospio cirrifera & 175 & Armandia polyophthalma & 84 & Scolelepis ciliata & 98 \\
\hline Cirratulus filiforms & 203 & Magelona papillocornis & 28 & Capitella capitata & 133 & Aonides oxycephala & 21 \\
\hline Capitella capitata & 28 & Cirratulus filiforms & 70 & Syllis gracilis & 119 & Polydora caeca & 1029 \\
\hline Owenia fusiformis & 21 & Armandia polyophthalma & 49 & Nephthys hombergii & 126 & Saccocirrus major & 56 \\
\hline Lepidonotus clava & 14 & Capitella capitata & 252 & Glycera convoluta & 84 & Syllis gracilis & 14 \\
\hline Syllis gracilis & 112 & Bispira volutacornis & 14 & Diopatra neapolitana & 28 & St.12 3m, ca.f.s. & No. \\
\hline Nereis irrorata & 35 & Lepidonotus clava & 98 & St.9 9m, f.s. & No. & Cirratulus filiforms & 63 \\
\hline Nephthys hombergii & 14 & Syllis gracilis & 21 & Oligochaeta & 280 & Armandia polyophthalma & 189 \\
\hline Onuphis eremita & 203 & Nereis irrorata & 49 & Polydora caeca & 28 & Capitella capitata & 126 \\
\hline $\begin{array}{ll}\text { St.2 } & 6 \mathrm{~m}, \mathrm{sl} .\end{array}$ & No. & Nephthys hombergii & 105 & Prionospio cirrifera & 119 & Nephthys hombergii & 63 \\
\hline Oligochaeta & 448 & Glycera convoluta & 42 & Cirratulus filiforms & 35 & St.13 22m, ca.f.s. & No. \\
\hline Polydora caeca & 364 & Goniada norvegica & 21 & Armandia polyophthalma & 21 & Oligochaeta & 63 \\
\hline Capitella capitata & 210 & Eunice vittata & 154 & Capitella capitata & 301 & Prionospio cirrifera & 116 \\
\hline Diopatra neapolitana & 70 & St.6 - $\quad 14 m$, c.s. & No. & Sabella pavonina & 14 & Capitella capitata & 63 \\
\hline St.3- $12 \mathrm{~m}$, sl. & No. & Oligochaeta & 56 & Polygordius neapolitanus & 217 & Saccocirrus major & 42 \\
\hline Oligochaeta & 34 & Polydora caeca & 21 & Eulalia viridis & 21 & Glycera convoluta & 31.5 \\
\hline Aonides oxycephala & 14 & Prionospio cirrifera & 168 & Syllis gracilis & 133 & St.14 2m, ca.f.s. & No. \\
\hline Capitella capitata & 63 & Capitella capitata & 28 & Glycera convoluta & 84 & Oligochaeta & 147 \\
\hline Cirratulus filiforms & 35 & Lepidonotus clava & 42 & Diopatra neapolitana & 77 & Aonides oxycephala & 28 \\
\hline Nephthys hombergii & 21 & Syllis gracilis & 63 & Lumbriconereis latreilli & 56 & Polydora caeca & 266 \\
\hline $\begin{array}{ll}\text { St.4 } & 10 \mathrm{~m}, \text { c.s. }\end{array}$ & No. & Nereis irrorata & 42 & $\begin{array}{ll}\text { St.10 } & 12 \mathrm{~m}, \text { f.s. }\end{array}$ & No. & Saccocirrus major & 154 \\
\hline Oligochaeta & 49 & Onuphis eremita & 35 & Oligochaeta & 98 & Glycera convoluta & 7 \\
\hline Prionospio cirrifera & 168 & Diopatra neapolitana & 14 & Polydora caeca & 56 & St.15 20m, ca.f.s. & No. \\
\hline Capitella capitata & 427 & Lumbriconereis latreilli & 28 & Prionospio cirrifera & 210 & Syllis gracilis & 105 \\
\hline Eulalia viridis & 21 & St.7 $3 m$, sl. & No. & Cirratulus filiforms & 21 & Nephthys hombergii & 84 \\
\hline Syllis gracilis & 28 & Oligochaeta & 202 & Armandia polyophthalma & 77 & St.16 $2 \mathrm{~m}$, ca.f.s. & No. \\
\hline Nereis irrorata & 329 & Magelona papillocornis & 16.8 & Capitella capitata & 140 & Oligochaeta & 101 \\
\hline Glycera convoluta & 77 & Capitella capitata & 118 & Sabella pavonina & 21 & Saccocirrus major & 25 \\
\hline Eunice vittata & 14 & Maupasia isochaeta & 8.4 & Polydontes maxilosus & 28 & & \\
\hline \multirow[t]{6}{*}{ Diopatra neapolitana } & 14 & Nereis irrorata & 806 & Syllis gracilis & 63 & & \\
\hline & & & & Nereis irrorata & 35 & & \\
\hline & & & & Nephthys hombergii & 28 & & \\
\hline & & & & Glycera convoluta & 84 & & \\
\hline & & & & Diopatra neapolitana & 28 & & \\
\hline & & & & Lumbriconereis latreilli & 42 & & \\
\hline
\end{tabular}



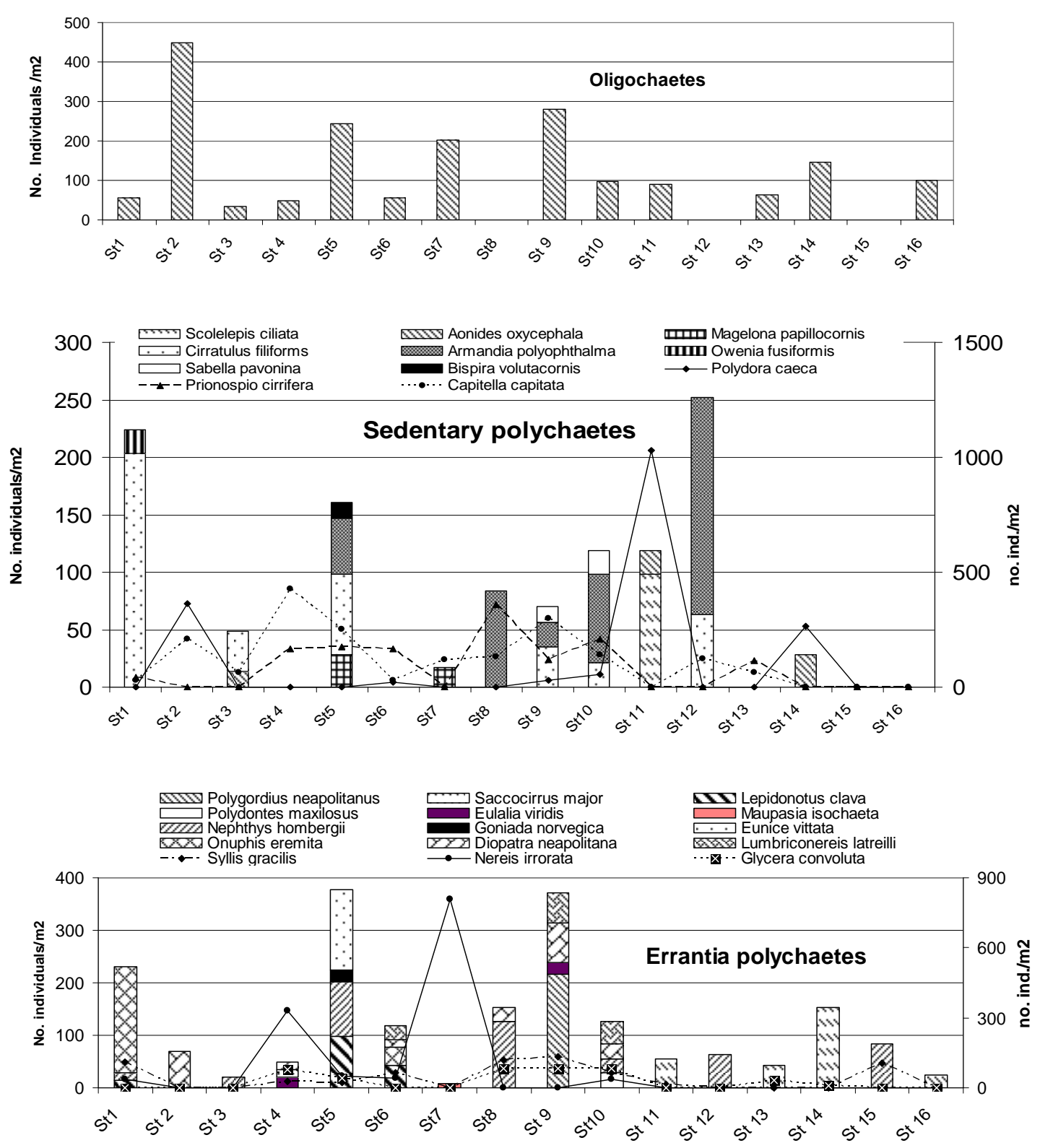

Fig. 3: Diversity in the average number of individuals of polychaetes (sedentary, Errantia) and oligochaetes during the study period.

Prionospis cirrifera, co-dominant with oligochaetes and Capitella capitata and was not appear at the area influenced by hydrocarbon pollutants (Sts. 11, 12). It was less frequent recorded during May (counting 588 at St.9) and in January (counting 126 and 588 ind. $/ \mathrm{m}^{2}$ at Sts. 8 and 10). On the other hand, it was predominant during March, July and November, reaching 714, 420, 126 and 84 ind. $/ \mathrm{m}^{2}$ respectively at Sts. 4, 5, 9 and 10. During July, its abundance varied between 210 to $462 \mathrm{ind} . / \mathrm{m}^{2}$ at Sts. 1, 4, 5, 6, and 13. It was more abundant at Sts. 6 and 8 during November that counting 789 and 2016 ind. $/ \mathrm{m}^{2}$ and its number was 252 and 588 ind. $/ \mathrm{m}^{2}$ at Sts. 5 and 10 respectively. So, it was apparently predominant in the stations close to the area with high stress of pollutants.

Nereis irrorata, also co-dominant with previous species, being recorded by large number in mesohaline area (St. 7). It was more frequent during May, being maximal 
at Sts. 4 and 7 counting 1296 and 4032 ind. $/ \mathrm{m}^{2}$ respectively, while its number ranged from 42 to 294 ind. $/ \mathrm{m}^{2}$ at Sts.1, 4-6 and 10. It was less frequent at Sts. 1 and 4 in March $\left(168,378\right.$ ind. $\left./ \mathrm{m}^{2}\right)$, at St.10 in September $\left(84\right.$ ind. $\left./ \mathrm{m}^{2}\right)$ and at St.6 in January $\left(126 \mathrm{ind} . / \mathrm{m}^{2}\right)$. In general, it did not appear in less stressed and tourist areas.

Sillys gracilis, recorded at different depths to $23 \mathrm{~m}$ and at different water quality; during May and November it was found only at St.9 counting 336 and 168 ind. $/ \mathrm{m}^{2}$ respectively and at St.6 (126 ind. $\left./ \mathrm{m}^{2}\right)$ in May. It was recorded at two stations during September, yielding 126 ind.$/ \mathrm{m}^{2}$ at St. 5 and $84 \mathrm{ind} . / \mathrm{m}^{2}$ at St.11. It was more frequent during July at Sts.8, 9 and 15, yielding 714, 294 and 210 ind. $/ \mathrm{m}^{2}$ and in January at Sts. $1,4,6$ and 10 reaching 168 to 672 ind. $/ \mathrm{m}^{2}$ respectively.

\section{Multi-regression analysis}

The relationship among the abiotic factors (organic matter, $\mathrm{Zn}, \mathrm{Pb}, \mathrm{Fe}, \mathrm{S} \% 0$, temperature) and the biotic variables (number of species, number of individuals, biomass, diversity indices) explored though multi-regression analysis were significant $(\mathrm{p}>0.05)$ as listed in Table (6).

Abundance of Oligochaeta was negative to $\mathrm{S} \% 0, \mathrm{pH}$, and positive to $\mathrm{NH}_{3}, \mathrm{NO}_{2}, \mathrm{PO}_{4}$, and $\mathrm{SiO} 4$ in seawater.

Abundance of Scolelepis was positive to $\mathrm{Fe}$ in seawater and $\mathrm{Mn}, \mathrm{Fe}, \mathrm{Cu}$ in sediment.

Abundance of Polydora was positive to Fe in seawater, $\mathrm{Mn}, \mathrm{Cu}$ in sediments.

Abundance of Prionospio was positive to temperature of seawater.

Abundance of Megelona was positive to $\mathrm{PO}_{4}, \mathrm{Mn}$ in seawater.

Abundance of Saccocirrus was positive to oxidisable organic matter OOM and total organic matter TOM.

Abundance of Lepodontus was positive to $\mathrm{Mn}$ in seawater.

Abundance of Maupasia was negative to temperature, $\mathrm{S} \% \mathrm{o}$, and positive to $\mathrm{NO}_{3}, \mathrm{PO}_{4}$, and $\mathrm{SiO}_{4}$ in seawater

Abundance of Syllis was positive to temperature of seawater and negative to $\mathrm{SiO}_{4}$ in seawater.

Abundance of Neries was negative to temperature, $\mathrm{S} \% 0$ and positive to $\mathrm{NO}_{3}, \mathrm{PO}_{4}$, and $\mathrm{SiO}_{4}$

Table 6: Correlations between oligochaetes and polychaetes species recorded in sediments samples at the different sampling sites during the period of collections, correlations are significant at $\mathrm{p}<.05000 \mathrm{~N}=16$

\begin{tabular}{|c|c|c|c|c|c|c|c|c|c|c|c|}
\hline $\begin{array}{l}\text { Param } \\
\text { eters }\end{array}$ & $\begin{array}{l}\text { Oligo } \\
\text { chaeta }\end{array}$ & $\begin{array}{l}\text { Scole } \\
\text { lepis }\end{array}$ & $\begin{array}{l}\text { Polyd } \\
\text { ora }\end{array}$ & $\begin{array}{l}\text { Prion } \\
\text { Ospio }\end{array}$ & $\begin{array}{l}\text { Mag } \\
\text { elona }\end{array}$ & $\begin{array}{l}\text { Sacco } \\
\text { cirrus }\end{array}$ & $\begin{array}{l}\text { Lepid } \\
\text { onotus }\end{array}$ & $\begin{array}{l}\text { Maup } \\
\text { asia }\end{array}$ & Syllis & Nereis & $\begin{array}{l}\text { Anoi } \\
\text { des }\end{array}$ \\
\hline Temp & & & & 0.52 & & & & -0.80 & 0.52 & -0.73 & \\
\hline $\mathrm{S} \% 0$ & -0.55 & & & & & & & -0.80 & & -0.76 & \\
\hline $\mathrm{PH}$ & -0.56 & & & & & & & & & & \\
\hline \multicolumn{12}{|l|}{ DO2 } \\
\hline NH3 & 0.71 & & & & & & & & & & \\
\hline $\mathrm{NO} 2$ & 0.71 & & & & & & & & & & \\
\hline NO3 & & & & & & & & 0.87 & & 0.82 & \\
\hline PO4 & 0.74 & & & & 0.51 & & & 0.61 & -0.53 & 0.55 & \\
\hline SIO4 & 0.66 & & & & & & & 0.57 & & 0.52 & \\
\hline OOM & & & & & & 0.86 & & & & & 0.72 \\
\hline TOC & & & & & & 0.55 & & & & & \\
\hline $\mathrm{MN}$ & & & & & 0.53 & & 0.59 & & & & \\
\hline FE & & 0.72 & 0.61 & & & & & & & & \\
\hline MN_SED & & 0.64 & 0.66 & & & & & & & & \\
\hline FE_SED & & 0.53 & & & & & & & & & \\
\hline CU_SED & & 0.59 & 0.67 & & & & & & & & \\
\hline
\end{tabular}


As revealed from Table (7) the values of diversity were significantly high only at Sts.6, 9, and 10, reaching 2.037 to 2.383 . For the Evenness values it was maximal at Sts. 5, 6, 9, and 10, being from 1.450 to 1.902 . The species richness was high $(>0.9)$ at Sts.3, 10, 12, 13, and 15. In general, the dropping in the diversity of polychaetes species was primarily attributed to the uneven distribution of individuals among the species. The general picture of the polychaetes community's diversity at the sampling sites by using the total average of species, indicating that the sites have high eutrophication parameters in sediments leading to high diversity in species and density.

Table 7: The values of diversity, Evenness, Richness index, number of species and average density of polychaetes and oligichaetes recorded at different sampling during the collection periods.

\begin{tabular}{|c|c|c|c|c|c|}
\hline Stations & $\begin{array}{l}\text { No. } \\
\text { species }\end{array}$ & $\begin{array}{l}\text { Abundance } \\
\text { No. ind. } / \mathrm{m}^{2}\end{array}$ & $\begin{array}{l}\text { Pielou's } \\
\text { Evenness } \\
\mathrm{J}^{\prime}=\mathrm{H}^{\prime} / \log \mathrm{S} \\
\end{array}$ & $\begin{array}{l}\text { Species richness } \\
\text { Margalef } \\
d=(\mathrm{S}-1) \log \mathrm{N}\end{array}$ & $\begin{array}{l}\text { Shannon-Weaver } \\
\text { index } \mathrm{H}^{\prime}=-\mathrm{i} \sum \mathrm{Pil} \log _{\mathrm{e}} \mathrm{Pi}\end{array}$ \\
\hline St.1 & 10 & 728 & 1.366 & 0.820 & 1.888 \\
\hline St. 2 & 4 & 1092 & 0.429 & 0.884 & 1.225 \\
\hline St.3 & 5 & 167 & 0.782 & 0.924 & 1.488 \\
\hline St.4 & 9 & 1127 & 1.138 & 0.731 & 1.606 \\
\hline St.5 & 14 & 1323 & 1.809 & 0.875 & 2.310 \\
\hline St.6 & 10 & 497 & 1.450 & 0.885 & 2.037 \\
\hline St.7 & 5 & 1151 & 0.568 & 0.550 & 0.885 \\
\hline St.8 & 7 & 931 & 0.878 & 0.883 & 1.719 \\
\hline St.9 & 13 & 1386 & 1.659 & 0.852 & 2.186 \\
\hline St.10 & 14 & 931 & 1.902 & 0.903 & 2.383 \\
\hline St.11 & 6 & 1309 & 0.697 & 0.457 & 0.818 \\
\hline St.12 & 4 & 441 & 0.493 & 0.921 & 1.277 \\
\hline St.13 & 5 & 315 & 0.695 & 0.939 & 1.511 \\
\hline St.14 & 5 & 602 & 0.625 & 0.776 & 1.248 \\
\hline St.15 & 2 & 189 & 0.191 & 0.991 & 0.687 \\
\hline St.16 & 2 & 109 & 0.213 & 0.779 & 0.540 \\
\hline
\end{tabular}

On the other hand, the estimated significant correlation for the oligochaetes and polychaetes species as variables $(\mathrm{p}>0.05)$ as listed in Table $(8)$ revealed that maximal correlations variables were recorded between Owenia fusiformis and Onuphis eremite; Bispira volutacornis and Lepidonotus clava; Bispira volutacornis and Eunice vittata.

Table 8: Significant correlation for the polychaetes and oligochaetes species as variables $(\mathrm{p}>0.05)$.

\begin{tabular}{|l|l|c|}
\hline \multicolumn{1}{|c|}{ Variable } & \multicolumn{1}{|c|}{ Variable } & Correlation \\
\hline Oligochaeta & Diopatra neapolitana & 0.66 \\
Magelona papillicornis & Goniada norvegica & 0.85 \\
Capitella capitata & Eulalia viridia & 0.77 \\
Capitella capitata & Glycera convoluta & 0.67 \\
Owenia fusiformis & Onuphis eremita & 0.99 \\
Sabella pavonina & Polygordius neapolitanus & 0.52 \\
Sabella pavonina & Lumbriconereis latreilli & 0.86 \\
Polygordius neapolitanus & Eulalia viridis & 0.68 \\
Bispira volutacornis & Lepidonotus clava & 0.91 \\
Bispira volutacornis & Eunice vittata & 0.99 \\
Lepidonotus clava & Goniada norvegica & 0.91 \\
Lepidonotus clava & Eunice vittata & 0.9 \\
Maupasia isochaeta. & Nereis irrorata & 0.92 \\
Goniada norvegica & Eunice vittata & 0.99 \\
\hline
\end{tabular}




\section{Abundance-Sites index Clustering}

Dendrograms based on Euclidean distances for clustering of 16 numerical density variables of polychaetes and oligochaetres collected at the different sites is shown in Fig. 4. The highest similarity is noticed between groups at Sts.8 and 4. The second high similarity is between stations 12 and 13, where the remaining sites show low similarity.

Cluster Hierarchical cluster analysis single linkage

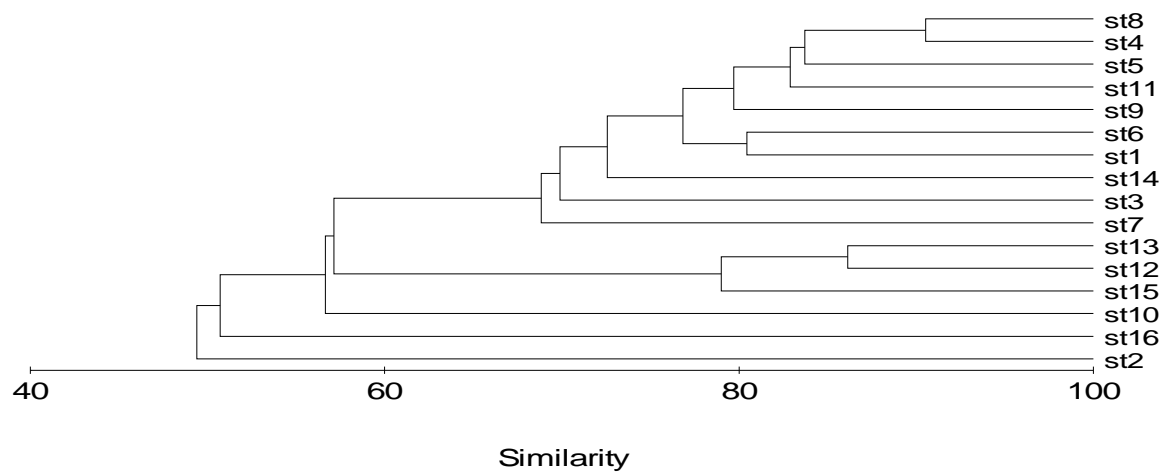

Fig. 4: Dedrogram for abundance of polychaetes and oligochaetes at sampling sites in the western coastal waters of Alexandria, during the period of study.

\section{DISCUSSION}

The sediments along the coast of Alexandria from the Western Harbor to El Agamy are slightly polluted by eutrophication parameters of the sediment. However, there are no indications of the benthos being affected by pollution along this stretch of the coast. The highest abundance and number of species are actually encountered in the sediment collected from inshore stations located in front of El Amom and Nobaria drains. This is probably due to the fact that the sediment there is more salty /clayey compared to all other study areas located to the west of Alexandria.

The area of El Mex Bay and El Nubouria estuary can be managed to be suitable for culture of marine organisms such as fish and mussels. Impact of human activities such as fishing, oil spills (SUMED area), heavy metals (untreated sewage discharge) and chemical residues (industrial wastes) outfalls is difficult to assess their influence on the benthic communities. High residue discharge can act as a source of natural eutrophication in shallow waters supporting filter feeding community composed of polychaetes, oligochaetes and molluscs in estuarine region off El Amom and El Nobaria drains. In the present work, an area with high trophic index of many polychaetes and oligochaetes species recorded in the vicinity of the outfall regions were significantly enriched in total number of individuals, total biomass and total number of species. Therefore, the influence of pollution could be varying on the benthic communities depending on the level of eutrophication and cleaning capacity of waters due to water circulation. The polychaetes and oligochaetes species found in contaminated area near the outfall have been reported as opportunistic species that can resist and live under more pollution stress. Herbivorous species can be introduced to control the growth of sea grass beds which act as a sediments trap and increasing the accumulation of pollutants on bottom sediments. 
Oligochaetes, many polychaetes species namely: Prionospis cirrifera, Cirratulus filiformis, Syllis gracilis., Glycera convolute, were recorded at most sites characteristic by high and moderate amount of pollution, while Nereis irrorata was recorded only at high stressed region. The total number of polychaetes species was higher at sites not close to outfall region which ranged from 9 to 14 species.

The occurrence of polychaete and oligochaete communities common in the stations close to increased pollutants outfalls namely Sts.2, 4 (El Amom drain), St.5 (El Dekhelah), Sts.7, 9 (El Nobaria drain) and St.11 (SUMED area). Their average frequencies ranged from $9 \%\left(1092 \mathrm{ind} . / \mathrm{m}^{2}\right)$ to $11 \%\left(1151 \mathrm{ind} . / \mathrm{m}^{2}\right)$ of the total number of polychaetes. Unfortunately, there are no available data for comparing with the present study to reveal the changes in the soft bottom communities of polychaetes. The remarkable changes were recorded among the sites and within the sites over the study period. Their abundance is greatly attributed to the presence of opertunitistic species, which are tolerant to enrichment of organic matter and chemical residues due to increased of human activities. The most common species considering the total average number of specimens per station were as follows: Capitella capitata $(15.4 \%)>$ Oligochaeta $(14.9 \%)>$ Polydora $(14.4 \%)>$ Prionospio $(11 \%)>$ Nereis $(10.6 \%)>$ Syllis $(5.4 \%)$.

Syllidae is one of polychaete families that inhabits hard bottom substrate (San Martin, 2003) and has been used as indicator taxon sensitive to pollution (Giangrande et al., 2004), while the opportunistic forms are rare as Syllis cf. hyallina. In this area of study particularly in the stressed area with pollutants from the two main drains high polychaete assemblages' revealed heavy modification. However, these were no reference data focused on the species composition and abundance of polychaetes relatively to the impact of environmental changes, the decreasing in number of individuals and species, in the western sites, was due to the low sedimentation rate and turbidity which typically indicates oligotrophic area.

As a whole, there were many opportunistic polychaete forms detected in the impacted site as far as the Capitellides assemblages, because species increasing in abundance showed an increase to that usually observed for typical opportunistic forms (Pearson and Rosenberg, 1978a,b). For most polychaete assemblages at the sampling sites, the observed trend was completely different. Syllid species seem to respond to disturbance rapidly with changes mainly occurring at shallow depths (completely disappeared in the impacted site in rocky shore habitat). El Mex Bay, an area highly influenced by human impacts had high density and number of polychaete species. The SUMED area, highly affected by hydrocarbon petroleum pollution (NIOF, 2006), showed the lowest number of species and abundance. The western area, which can be influenced by the impact of pollutants (due to the direction of current, mainly from the west to the east, but the area showed the lowest number of species and abundance. The increase in polychaete abundance observed in outfall area could be due to the effect of trophic level. A similar effect was reported at the Eastern Harbour of Alexandria (by the author, unpublished data), suggesting that high intensity of polychaetes could be due to the effect of eutrophication of the area. Syllidae were reported as a biogeographic and bioclimatic indicator within the Mediterranean basin (Musco, and Giangrande, in press) with distinct distribution that mainly follows a bioclimatic (ecological) rather than biogeographical pattern. Regular data of polychaetes are suitable to be a considered indicator needed to compare the present situation with previous one to be able to document changes in biodiversity and the ecosystem of communities. 
Warwick and Clarke (1991) and Clarke and Warwick, (1998) introduced the taxonomic distinctness index (TD) based on the taxonomic relatedness of species in a given sample. The TD decreased with increasing stress (Warwick and Clarke, 1991). This means that a method to quantify biodiversity becomes a method to measure stress, to be used for monitoring and ecological analysis. Some area habitats (lagoons, soft bottom, and hard bottom) were compared in number of polychaetes families and species often by computation of the ratio of species/families. Comparing soft bottom areas along the Tyrrhenian coast submitted to different trophic input from rivers (Gambi and Giangrande, 1986), the ratio was higher, ranging from 3.5 to 2.1. In this case, the family level leads to a loss of information. However, the highest ratio was observed for hard bottom environment which often are colonized by a great number of closely related species. On hard bottom, values ranged from 6.4 to 4.6 and result quite similar comparing different areas with similar environmental conditions but located in different basins (Giangrande, 1988; Giangrande et al., 2003). The decrease of the species/family ratio was related with increasing pollution coming from the comparison of different hard bottom polychaete assemblages from the same geographical area, but related to different human impacts. The conclusion from the present analysis is that only the brackish waters are the environments where TD can be applied.

Under conditions of intermediate disturbance, the relative abundance of the species rather than the species composition was changed. This indicated that the impact of human activities on the benthic communities would be very difficult to assess. However, the data show that a shallow environment with a dense eutrophical level supports a natural feeding source for those filter feeders, suspension feeders, deposit feeders and herbivores. These species were reported as filter feeding community that can be used as a natural eutrophication control besides filter feeding community of molluscs and demersal fishes.

Daucer and Conner (1980) reported that polychaete populations of an estuarine, intertidal habitat in the vicinity of a sewage outfall were significantly enriched in total number of individuals, total biomass and total number of species. At stations near outfall of the two main drains (El Amom, Nobaria) as polluted bottoms with organic matter, heavy metals and nutrients were dominated by many polychaete species during the study period. Warwick and Clarke (1991) and Clarke and Warwick, (1998) introduced the taxonomic distinctness index (TD) based on the taxonomic relatedness of the species in a given sample. Quadros et al. (2009) studied monthly Polychaete assemblages and associated environment on intertidal stations along the extremely polluted west coast of India and compared with past available data to investigate changes in the creek ecology due to various anthropogenic activities like industrial, domestic, and solid waste disposal along with land reclamation. Ceratonereis burmensis and Lycastis indica were the most abundant and omnipresent polychaetes in the creek, indicating their tolerance and adaptability to various degrees of pollution. They found that species richness was correlated positively with clay and negatively with silt. Hydro-sedimentological investigations revealed enhancement of total nitrogen (TN) and organic carbon (Corg) load and hypoxic levels of dissolved oxygen (DO) over the years. Silt component of sediment was increasing, with proportionate decrease in clay due to various anthropogenic disturbances. 


\section{REFERENCES}

Borja, A. and Muxika, I. (2005). Do benthic tools respond to all impact sources? The Case of AMBI (AZTI Marine Biotic Index). In indicators of stress in the marine benthos, UNESCO, Intergovernmental Oceanographic Commission Workshop Report No. 195

Boyd, S.E.; Cooper, K.M.; Limpenny, D.S.; Kilbride, R.; Rees, H.I.; Dearnaley, M.P.; Stevenson, J.; Medows, W.J. and Morris, C. D. (2004). Assessment of the rehabilitation of the seabed following marine dredging. Science Series Technical Report. CEFAS Lowestoft, No. 121: 154 pp.

Bray, J. R. and Curtis, J. T. (1957). An ordination of the upland forest communities of the Southern Wisconsin. Ecol. Monogr., 27: 325-349.

Clarke, K.R. and Gorley, R.N. (2001). PRIMER V.5 User Manual Tutorial. PRIMERE Ltd. Plymouth, 91 pp.

Clarke, K.R. and Warwick, R. M. (1998). A taxonomic distinctness index and its statistical properties. J. Applied Ecol., 35: 523-531.

Cray, J. S. and Mirza, F.B. (1979). A possible method for the detection of disturbance on marine benthic communities. Mar.Poll.Bull., 10: 142-146.

Daucer, D. M. and Conner, W.G. (1980). Effects of moderate sewage input on benthic polychaete populations. Est. Coast. Mar. Sci., 10: 335-346.

Desprez, M. (2000). Physical and biological impact of marine aggregate extraction along the French Coast of the Eastern English Channel: short and long-term post-dredging restoration. ICES J. Mar. Sci., 57: 1428-1438.

Fauvel, P. (1923). Faune de France, V. Polychetes errantes. Le Chevalier, Paris, $488 \mathrm{pp}$.

Fauvel, P. (1927). Faune de France, 16. Polychetes sedentaires. Addenda aux errantes archiannelides myzostomaires: $494 \mathrm{pp}$.

Fauvel, P. (1937). Les fonds de peches pres d'Alexandrie, XI, Annelides polychetes. Notes Mem. Dir. Rech. Pech., 19: 1-60.

Gambi, M.C. and Giangrande, A. (1986). Distribution of soft-bottom Polychaetes in two coastal areas of the Tyrrhenian Sea (Italy): structural analysis. Estu. Coast. Shelf Sci., 23: 847-862.

Giangrande, A. (1988). Polychaete zonation and its relation to algal distribution down a vertical cliff in the western Mediterranean (Italy); a structural analysis. J. Exper. Mar. Biol, Ecol., 120: 263-276.

Giangrande, A.; Delos, A.I.; Fraschetu, S.; Musco, L.; Licciano, M. and Terlizzi, A. (2003). Polychaete assemblages of rocky-shores along the South Adriatic 
coast (Mediterranean Sea): pattern of spatial distribution. Mar. Biol., 143: 1109-1116.

Giangrande, A.; Delos, A.L.; Musco, L.; Licciano, M. and Pierri, C. (2004). Polychaete assemblages of rocky shore along the South Adriatic coast (Mediterranean Sea). Cah. de Biol. Mar., 45: 85-95.

ICES (2001). Effects of Marine Sediment on the Marine Ecosystem. ICES Cooperative Research Report No. 247.

Jones, G. and Candy, S. (1981). Effects of dredging on the macrobenthic infauns of Botany Bay. Aust. J. Mar. Freshw. Res., 32:379-398.

Kenny, A. J. and Rees, H. I. (1996). The effects of Marine gravel extraction on the macrobenthos: results 2 years post-dredging. Mar. Poll. Bull., 32: 615-622.

Laetz, C. (1998). Marine Benthic Invertebrate Communities Near King County's Wastewater Outfalls. Puget Sound Research, 754-759.

Musco, L., Giangrande, A., in press. Mediterranean Syllidae (Annelida: Polychaeta) revisited: biogeography, diversity and species fidelity to environmental features. Mar.Ecol. Series.

Newell, R.C.; Seiderer, L.J.; Simpson, N.M.; Robinson, J.E. (1998). Impacts of Marine Aggregate dredging on benthic macrofauna off the south coast of the United Kingdom. J. Coast. Res., 20: 115-125.

NIOF (2006). Final Report on Effects of Industrial, Tourist Activities and Marine Transport on Physical, Chemical and Biological Characteristics of water and Fish Populations West of Alexandria

Pearson, T. H. and Rosenberg, R. (1978a). Macrobenthic succession in relation to organic enrichment and pollution of the marine environment. Oceanogr. Mar. Biol. Ann. Rev., 16: 229-311.

Pearson, T.H. and Rosenberg, R.(1978b). A Comparative study of the effects on the Marine Environment of Wastes from Cellulose Industries in Scotland and Sweden. Ambio, 5: 77-79.

PTI, (1993). Recommendations for Assessing Adverse Benthic Effects in Puget Sound. Prepared for: Washington State Department of Ecology Sediment Management Unit, Olympia, WA. Quintino, V., M.

Quadros G.; Sukumaran, S. and Athalye, R. P. (2009). Impact of the changing ecology on intertidal polychaetes in an anthropogenically stressed tropical creek, India. Aquat. Ecol. 43:977-985.

Rees, H.L.; Sneddon, J. and Boyd, S.E. (2005). Benthic indicators: criteria for evaluation Scientific and Management Effectiveness. In Indicators of Stress in the Marine Benthos, UNESCO, Intergovernmental Oceanographic Commission Workshop Report No. 195 
Rumohr, H. (2005). A 5-Step Succession Model for the Baltic - A Future Management Tool? In Indicators of Stress in the Marine Benthos, UNESCO, Intergovernmental Oceanographic Commission Workshop Report No. 195

San Martin, G. (2003). Annelids. Polychaeta II: Syllidae. In: Ramos, M.A. et al. (Eds.), Fauna, Musco National de Ciencias Naturales. Vol. 21. CSIC, Madrid, pp. 1-529.

Shine, J. (2005). A Summary of Results if IOC-BIG Benthic-TOC Study. In Indicators of Stress in the Marine Benthos, UNESCO, Intergovernmental Oceanographic Commission Workshop Report No. 195.

Steur, A., (1935). The fishery grounds near Alexandria. Notes and Memoirs, 8: 18 pp.

Warwick R.M., Clarke K.R. (1991). A comparison of some methods for analyzing changes in benthic community structure. J. Mar. biol. Ass. U.K., 71: 225-244.

\section{ARABIC SUMMARY}

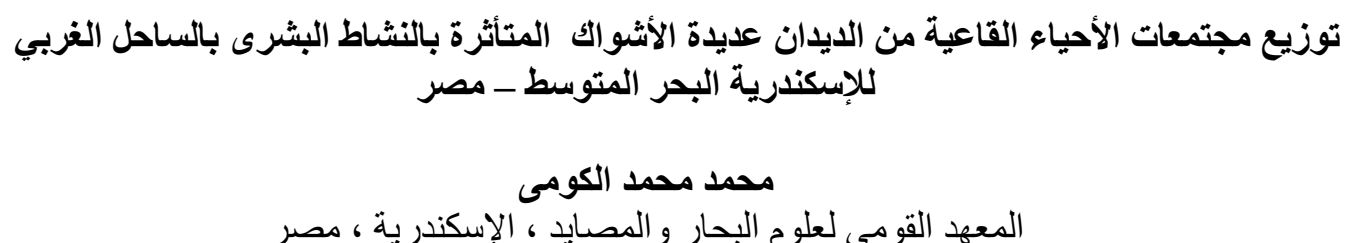

Komimohamed50@yahoo.com

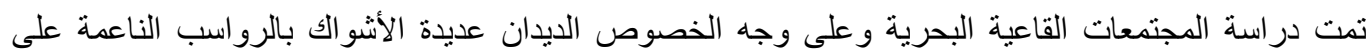



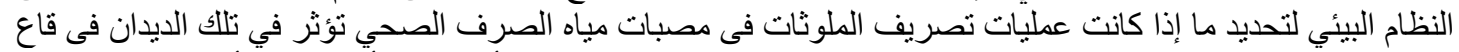

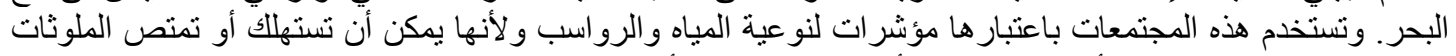

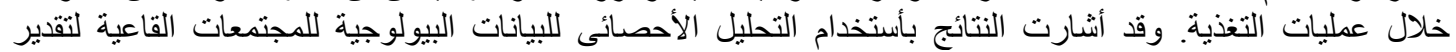

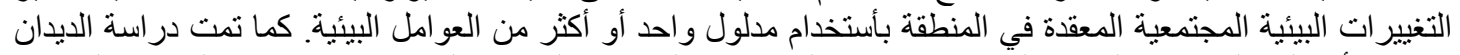

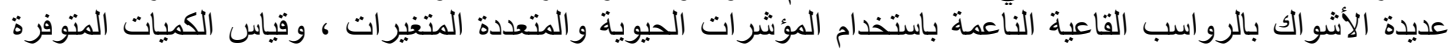

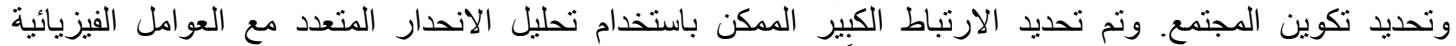

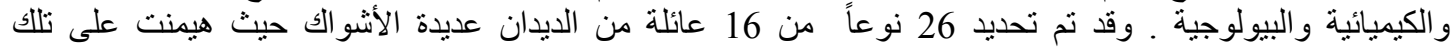

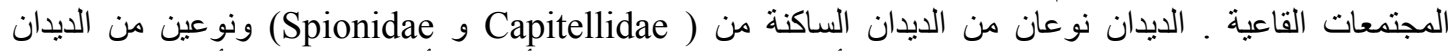

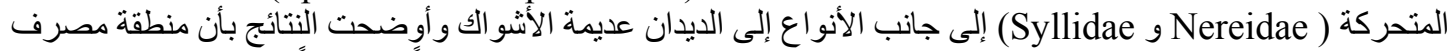

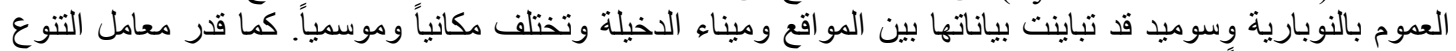

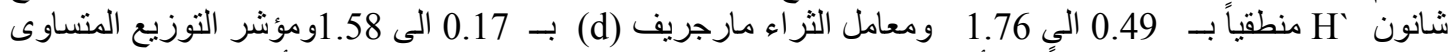

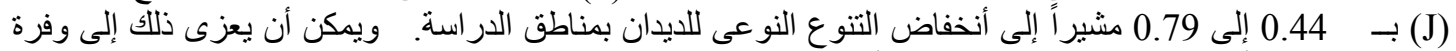

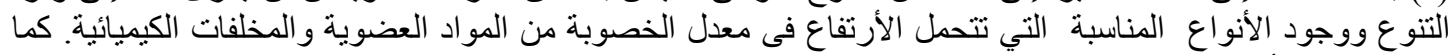

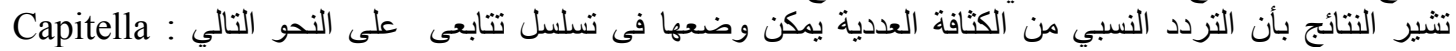
$(15.4 \%)>$ Oligochaeta (14.9\%) > Polydora (14.4\%) > Prionospio (11\%) > Nereis (10.6\%) >

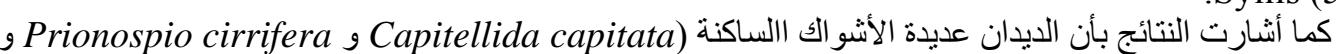
.Syllis $(5.4 \%)$

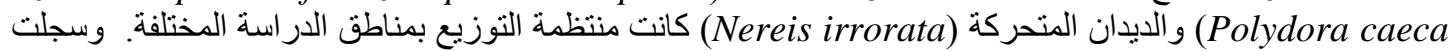

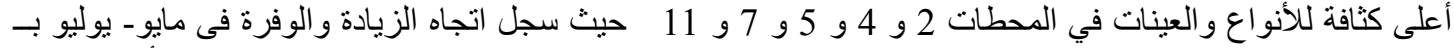
1092 إلى 1385 كائن/م2 و وتلك الزيادة في الوفرة العددية كانت في منطقة المصبات ويرجع ذلك إلى تأثنير مستوى 